\title{
Radiologic features of pneumonitis associated with nivolumab in non-small-cell lung cancer and malignant melanoma
}

\author{
Tomohisa Baba*,1, Fumikazu Sakai², Terufumi Kato ${ }^{3}$, Masahiko Kusumoto $^{4}$, \\ Hirotsugu Kenmotsu ${ }^{5}$, Hiroaki Sugiura ${ }^{6}$, Junya Tominaga7, Katsunori Oikado ${ }^{7}$, \\ Masafumi Sata ${ }^{9}$, Masahiro Endo ${ }^{5}$, Noriyo Yanagawa ${ }^{10}$, Shinichi Sasaki ${ }^{11}$, Tae Iwasawa ${ }^{1}$, \\ Yoshinobu Saito ${ }^{12}$, Yutaka Fujiwara ${ }^{13}$, Yuichiro Ohe ${ }^{4}$, Naoya Yamazaki $^{4}$ \\ Takahiko Sakamoto ${ }^{14}$, Takashi Koshiba ${ }^{14}$ \& Kazuyoshi Kuwano ${ }^{15}$ \\ ${ }^{1}$ Kanagawa Cardiovascular \& Respiratory Center, Yokohama, Japan \\ ${ }^{2}$ Saitama Medical University International Medical Center, Hidaka, Japan \\ ${ }^{3}$ Kanagawa Cancer Center, Yokohama, Japan \\ ${ }^{4}$ National Cancer Center Hospital, Tokyo, Japan \\ ${ }^{5}$ Shizuoka Cancer Center, Nagaizumi, Japan \\ ${ }^{6}$ Keio University School of Medicine, Tokyo, Japan \\ ${ }^{7}$ Tohoku University School of Medicine, Sendai, Japan \\ ${ }^{8}$ Cancer Institute Hospital, Tokyo, Japan \\ ${ }^{9}$ Jichi Medical University, Tochigi, Japan \\ ${ }^{10}$ Tokyo Metropolitan Cancer \& Infectious Diseases Center Komagome Hospital, Tokyo, Japan \\ ${ }^{11}$ Juntendo University Urayasu Hospital, Urayasu, Japan \\ ${ }^{12}$ Nippon Medical School Hospital, Tokyo, Japan \\ ${ }^{13}$ Mitsui Memorial Hospital, Tokyo Japan \\ ${ }^{14}$ Ono Pharmaceutical Co., Ltd, Osaka, Japan \\ ${ }^{15}$ The Jikei University School of Medicine, Tokyo, Japan \\ *Author for correspondence: Tel.: +81 457019 581; baba@kanagawa-junko.jp
}

\begin{abstract}
Aim: To assess the clinical features/imaging characteristics of pneumonitis reported during nationwide nivolumab postmarketing surveillance in Japan. Patients \& methods: Clinical and radiological data were collected from pneumonitis cases reported during/after nivolumab treatment for melanoma or non-smallcell lung cancer. The expert central review committee evaluated each case. Results: Among 144 cases analyzed, 91 (63.2\%) had radiological patterns considered typical for drug-induced pneumonitis and 53 $(36.8 \%)$ patients had previously unobserved patterns with one or more atypical features, including 23 cases $(16.0 \%)$ with ground glass opacity confined to the area around the tumor (peritumoral infiltration). A higher proportion of patients with (vs without) peritumoral infiltration had an antitumor response to nivolumab. Conclusion: Images of nivolumab-induced pneumonitis showed previously unobserved radiological patterns.
\end{abstract}

First draft submitted: 21 February 2019; Accepted for publication: 9 April 2019; Published online:

25 April 2019

Keywords: immune checkpoint inhibitor $\bullet$ interstitial lung disease $\bullet$ nivolumab $\bullet$ pneumonitis $\bullet$ PD-1 inhibitor

Therapeutic interventions for the treatment of cancer, including conventional chemotherapeutic drugs and molecular-targeted agents, have the potential to cause interstitial lung disease (ILD) or pneumonitis; moleculartargeted agents are among the most common ILD-inducing drugs [1,2]. Patients with drug-induced ILD can present precipitously with acute diffuse alveolar damage (DAD), which can be fatal [1].

Nivolumab, a fully human IgG4 monoclonal antibody, targets PD-1 (PD-1), one of the T-cell surface membrane receptors [3]. Nivolumab was first approved in Japan in 2014 for the treatment of unresectable malignant melanoma; given its demonstrated antitumor activity, nivolumab is also used widely for other malignancies, including lung cancer [3,4]. Nivolumab-induced pneumonitis was reported in 4.6\% (6/131) and 3.5\% (10/287) of patients, respectively, in the Phase III CheckMate 017 and CheckMate 057 trials in patients with non-small-cell lung cancer 
(NSCLC) [3,4]. This is consistent with rates of 3.6 and $4.1 \%$ reported for meta-analyses of PD-1 inhibitors in NSCLC $[5,6]$.

Recently, a new type of pneumonitis associated with PD-1 axis inhibitors has been reported as peritumoral inflammation in some patients [7]. Kato et al. analyzed the clinical and radiological features of nivolumab-associated pneumonitis in Phase II clinical trials [8]; and Naidoo et al. reported pneumonitis in patients treated with antiPD-1/PD-L1 therapy [9]. However, these patients account for only small numbers from clinical trials without comorbidities and with good performance status. With such small patient counts within clinical trials, there was consensus that a larger real-world study could provide additional information. Therefore, we analyzed the clinical features and imaging characteristics of pneumonitis reported during a nationwide postmarketing surveillance (PMS) program of nivolumab in Japan.

\section{Methods}

Cases reported by physicians as ILD or pneumonitis during or after nivolumab treatment for melanoma or NSCLC through the end of November 2016 were identified from nationwide PMS data on nivolumab in Japan. The study complied with Japanese Good Post-Marketing Study Practice. Clinical and radiological data were obtained for each patient. For inclusion in the analysis, patients had to have evaluable computed tomography (CT) data available from the time of pneumonitis onset. Patients who had received an epidermal growth factor receptor-tyrosine kinase inhibitor (EGFR-TKI) after nivolumab were excluded, in order to rule out EGFR-TKI-induced ILD. The ILD Expert Central Review Committee (ECRC) consisted of eight chest radiologists, four pulmonologists specializing in diffuse lung disease and four pulmonary oncologists.

For each case, using pretreatment CT data, two radiologists independently evaluated the presence of interstitial pneumonia (IP) and honeycombing, level of emphysema, percent of normal lung field and preceding radiation pneumonitis/fibrosis. Using CT data at the time of ILD onset, the radiologists evaluated the presence and distribution of ground glass opacity (GGO), consolidation, reticulation and traction bronchiectasis, and determined the validity of ILD in new diffuse infiltration. CT patterns of ILD were classified, according to the ATS/ERS international multidisciplinary classification of IP, as acute interstitial pneumonia (AIP)/DAD-like pattern, hypersensitivity pneumonia (HP)-like pattern, cryptogenic organizing pneumonia (COP)-like pattern, nonspecific interstitial pneumonia (NSIP)-like pattern or others [10]. Using CT data at follow-up, the radiologists evaluated the outcome of ILD and determined the nivolumab antitumor effect. Two pulmonologists/pulmonary oncologists independently determined the validity of ILD, based on symptoms and physical findings, clinical course, laboratory data (including WBC, CRP, LDH, KL-6, BNP, CEA and others), results of bacteriological examination and findings from bronchoalveolar lavage (BAL). After independent evaluation by the radiologists, pulmonologists and pulmonary oncologists, the ECRC discussed the results and determined the validity of ILD after reaching consensus among all members.

A 'typical' pattern of pneumonitis images referred to findings similar to those commonly appearing during treatment with conventional chemotherapy or molecular-targeted drugs: GGO or consolidation showing nonsegmental distribution bilaterally or dominant in the lung contralateral to the tumor [11]. Initial evaluation by the ECRC found some atypical pneumonitis cases which were also added to the evaluation. 'Atypical' features described: GGO confined to the area around the tumor (peritumoral infiltration; PTI); GGO or consolidation around radiation fibrosis consistent with the radiation field (exacerbation of radiation fibrosis); diffuse pulmonary infiltration radiologically resembling IP with exacerbation of subclinical infection (intensified infections); and abnormal opacities largely confined to the lung ipsilateral to the tumor (ipsilateral to the tumor).

Data were summarized using number of patients and percentages, and mean \pm standard deviation (SD) values.

\section{Results}

A comprehensive review of 195 pneumonitis cases was completed by 27 November 2016 and the analysis of these cases is reported here.

A causal relationship of pneumonitis with nivolumab could not be ruled out for 160 of the 195 reviewed cases. Of these, 144 patients with CT data available at pneumonitis onset and not receiving an EGFR-TKI after nivolumab, were included in the analysis (Figure 1). The general characteristics of patients with pneumonitis are summarized in Table 1 . The mean age of the population was 66.6 years, the majority $(82.6 \%)$ were male and most $(89.6 \%)$ had NSCLC. Most patients (79.2\%) had received 1-3 treatment lines and $42.4 \%$ had previously received radiotherapy. The mean time to ILD after the first dose of nivolumab was $47.3 \pm 64.4$ days. 


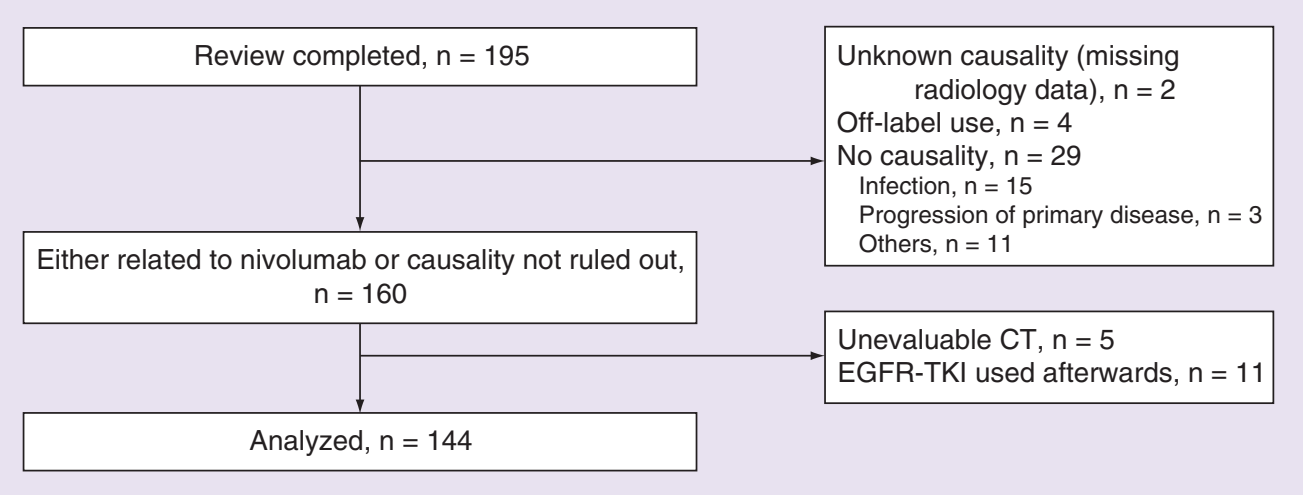

Figure 1. Flow diagram of pneumonitis cases.

EGFR-TKI: Epidermal growth factor receptor-tyrosine kinase inhibitor.

\section{Table 1. Patient characteristics.}

\begin{tabular}{|c|c|c|c|c|}
\hline Characteristic & Total N = 144 & PTI N = 23 & Non-PTI N = 121 & $\begin{array}{l}\text { Significant difference } \\
\text { (p-value) }\end{array}$ \\
\hline Age (years) & $66.6 \pm 9.47$ & $66.6 \pm 8.64$ & $66.6 \pm 9.65$ & N.S. $(0.9980)^{t}$ \\
\hline $\begin{array}{l}\text { Sex } \\
\text { - Male } \\
\text { - Female }\end{array}$ & $\begin{array}{l}119(82.6) \\
25(17.4)\end{array}$ & $\begin{array}{l}19(82.6) \\
4(17.4)\end{array}$ & $\begin{array}{l}100(82.6) \\
21(17.4)\end{array}$ & $\begin{array}{l}\text { N.S. } \\
(0.9967) \times\end{array}$ \\
\hline $\begin{array}{l}\text { Smoker } \\
\text { - None } \\
\text { - Former } \\
\text { - Current }\end{array}$ & $\begin{array}{l}29(20.1) \\
103(71.5) \\
12(8.3)\end{array}$ & $\begin{array}{l}3(13.0) \\
19(82.6) \\
1(4.3)\end{array}$ & $\begin{array}{l}26(21.5) \\
84(69.4) \\
11(9.1)\end{array}$ & $\begin{array}{l}\text { N.S. } \\
(0.4326) \times\end{array}$ \\
\hline $\begin{array}{l}\text { Cancer type } \\
\text { - Advanced NSCLC } \\
\text { - Adenocarcinoma } \\
\text { - Squamous cell carcinoma } \\
\text { - Other/unknown } \\
\text { - Malignant melanoma }\end{array}$ & $\begin{array}{l}129(89.6) \\
61(47.3)^{\dagger} \\
52(40.3)^{\dagger} \\
16(12.4)^{\dagger} \\
15(10.4)\end{array}$ & $\begin{array}{l}23(100) \\
13(56.5)^{\dagger} \\
8(34.8)^{\dagger} \\
2(8.7)^{\dagger} \\
0(0)\end{array}$ & $\begin{array}{l}106(87.6) \\
48(45.3)^{\dagger} \\
44(41.5)^{\dagger} \\
14(13.2)^{\dagger} \\
15(12.4)\end{array}$ & - \\
\hline $\begin{array}{l}\text { Treatment line } \\
-0-1 \\
-2-3 \\
-4-5 \\
-\geq 6 \\
- \text { Unknown }\end{array}$ & $\begin{array}{l}58(40.3) \\
56(38.9) \\
18(12.5) \\
10(6.9) \\
2(1.4)\end{array}$ & $\begin{array}{l}8(34.8) \\
11(47.8) \\
2(8.7) \\
1(4.3) \\
1(4.3)\end{array}$ & $\begin{array}{l}50(41.3) \\
45(37.2) \\
16(13.2) \\
9(7.4) \\
1(0.8)\end{array}$ & $\begin{array}{l}\text { N.S. } \\
(0.9976)^{w}\end{array}$ \\
\hline Previous radiation & $61(42.4)$ & $13(56.5)$ & $48(39.7)$ & $\begin{array}{l}\text { N.S. } \\
(0.1338) \times\end{array}$ \\
\hline $\begin{array}{l}\text { CTCAE grade of ILD } \\
-1-2 \\
-3-5 \\
- \text { Unknown }\end{array}$ & $\begin{array}{l}48(33.3) \\
56(38.9) \\
40(27.8)\end{array}$ & $\begin{array}{l}9(39.1) \\
5(21.7) \\
9(39.1)\end{array}$ & $\begin{array}{l}39(32.2) \\
51(42.1) \\
31(25.6)\end{array}$ & $\begin{array}{l}\text { N.S. } \\
(0.1470)^{w}\end{array}$ \\
\hline Days to ILD after initial dose of nivolumab & $47.3 \pm 64.7$ & $32.9 \pm 29.7$ & $50.2 \pm 69.3$ & $\begin{array}{l}\text { N.S. } \\
(0.2418)^{t}\end{array}$ \\
\hline \multicolumn{5}{|c|}{$\begin{array}{l}\text { Data are } n(\%) \text { or mean } \pm \text { SD. } \\
\text { †Data are expressed as a percentage of all NSCLC cases. } \\
\text { t: } t \text {-test; } w \text { : Wilcoxon rank-sum test; } X: \chi^{2} \text { test. } \\
\text { CTCAE: Common Terminology Criteria for Adverse Event; ILD: Interstitial lung disease; N.S.: Not statistically significant; NSCLC: Non-small-cell lung cancer; PTI: Peritumoral } \\
\text { infiltration. }\end{array}$} \\
\hline
\end{tabular}

Among the 144 cases analyzed, 91 (63.2\%) had radiological patterns that were considered typical for druginduced pneumonitis associated with chemotherapy or molecular-targeted drugs, while $53(36.8 \%)$ had patterns that included one or more atypical features. Among patients with atypical features, 23 (16.0\%) had PTI, four (2.8\%) had signs of intensified infection (one case each of nontuberculous mycobacteria and Pseudomonas aeruginosa and two cases of pneumocystis pneumonia [PCP]), 23 (16.0\%) had exacerbation of radiation fibrosis and 23 (16.0\%) had opacities confined to the lung ipsilateral to the tumor. Figure 2 shows CT images illustrating the range of observed pneumonitis patterns. 
(A1)

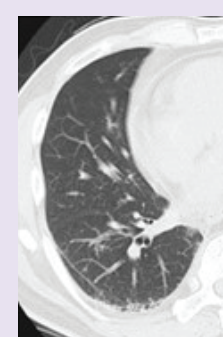

(B1)

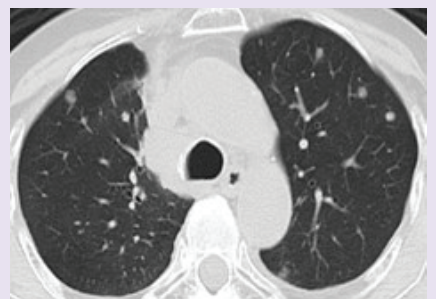

(c1)

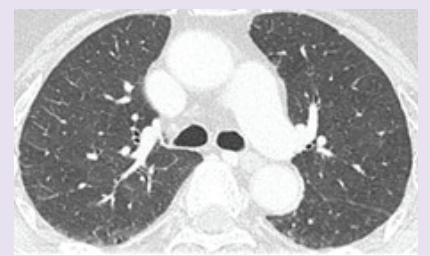

(D1)

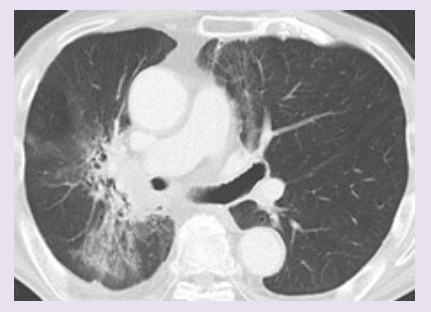

(E1)
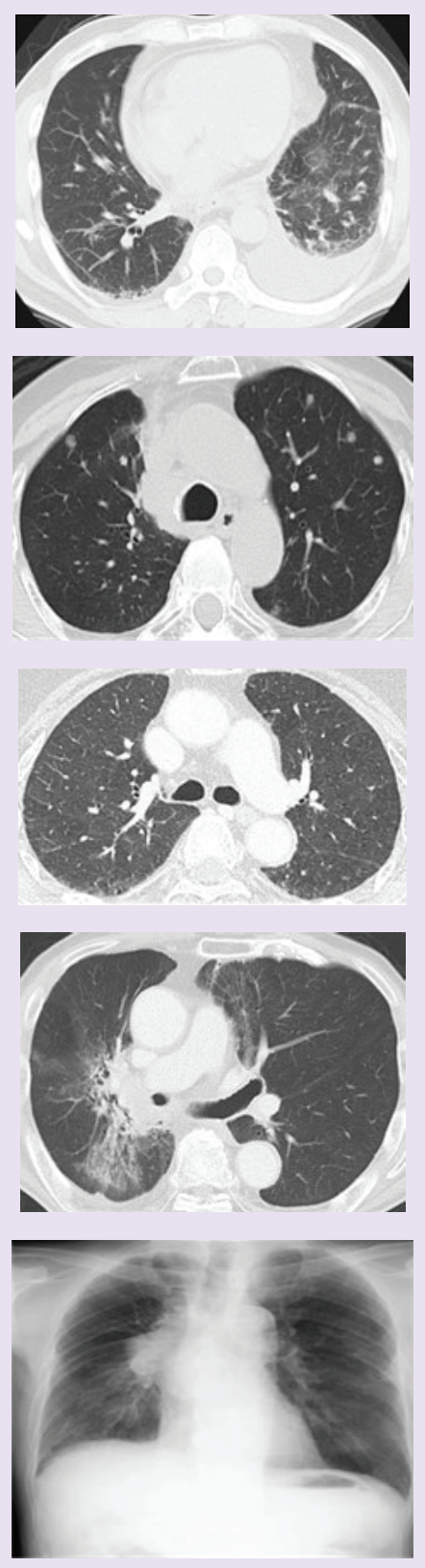
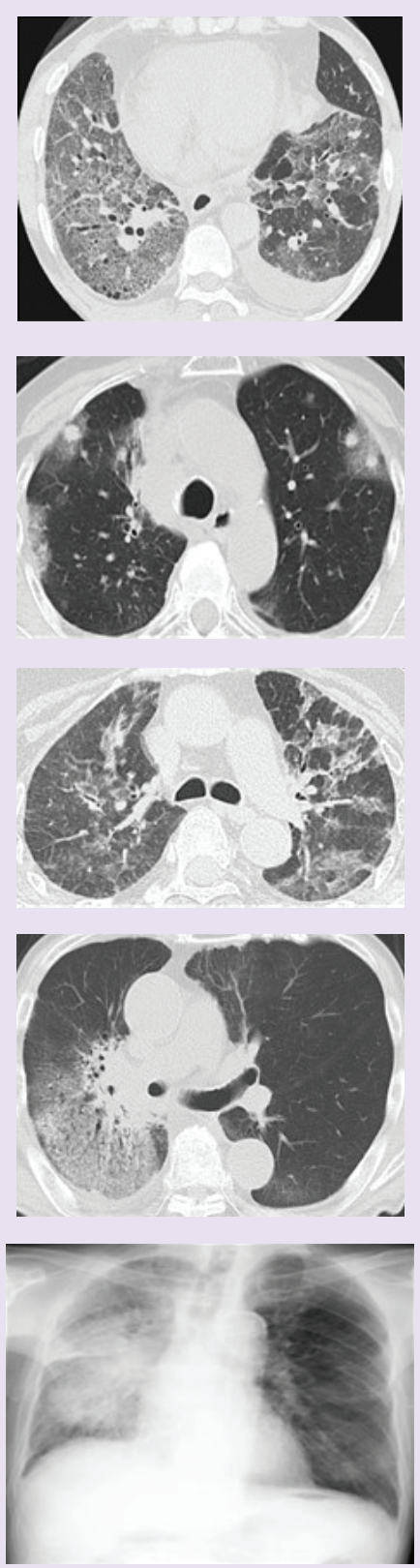

(A2)

(B2)

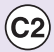

(D2)
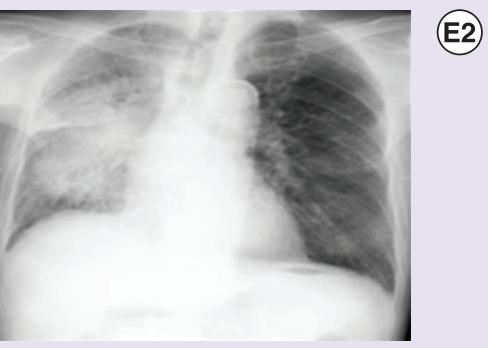

Figure 2. Representative radiological manifestations of nivolumab-associated pneumonitis. (A1), (B1), (C1), (D1), (E1), (F1) are CT images before nivolumab treatment. (A2), (B2), (C2), (D2), (E2), (F2) are CT images at the onset of pneumonitis. (A1) and (A2), pneumonitis with AIP/DAD like pattern in a 57-year-old male with squamous lung cell carcinoma. Ground glass opacities (GGO) appeared in almost all the lung field. Distorted interlobular septa, pleura and vessels indicated diffuse alveolar damage. (B1) and (B2), peritumoral infiltration (PTI) in a 70-year-old female with lung adenocarcinoma. GGO surrounding tumors, so-called PTI, appeared after nivolumab treatment. (C1) and (C2), pattern similar to intensified infection, in a 72-year-old female with melanoma. Onset of interstitial lung disease occurred during the night of the day of the first dose of nivolumab. GGO with a mosaic appearance before treatment, and high beta-D-glucan level $(157 \mathrm{pg} / \mathrm{ml})$ at the onset of pneumonitis, suggested pneumocystis pneumonia. This case resulted in a fatal outcome. (D1) and (D2), pattern similar to exacerbation of radiation fibrosis. CT scan before nivolumab treatment was 50 months after the completion of right hilar radiotherapy. Nivolumab reactivated previous radiation fibrosis. (E1) and (E2), infiltration in the ipsilateral lung field in a 74-year-old male with squamous cell lung carcinoma. Consolidation appeared in the ipsilateral lung field with right lung carcinoma. 
Table 2. Chest computed tomography findings before nivolumab treatment and at the time of onset of nivolumab-associated interstitial lung disease, and the pattern of interstitial lung disease.

\begin{tabular}{|c|c|c|c|c|}
\hline & Total $N=144$ & PTI N = 23 & Non-PTI N = 121 & $\begin{array}{l}\text { Significant difference } \\
\text { (p-value) }\end{array}$ \\
\hline \multicolumn{5}{|l|}{ CT findings before nivolumab treatment } \\
\hline \multicolumn{5}{|l|}{ Interstitial pneumonia } \\
\hline $\begin{array}{l}\text { None } \\
\text { - Mild } \\
\text { - Moderate } \\
\text { - Severe } \\
\text { - Unknown }\end{array}$ & $\begin{array}{l}92(63.9) \\
35(24.3) \\
9(6.3) \\
3(2.1) \\
5(3.5)\end{array}$ & $\begin{array}{l}21(91.3) \\
1(4.3) \\
0(0) \\
0(0) \\
1(4.3)\end{array}$ & $\begin{array}{l}71(58.7) \\
34(28.1) \\
9(7.4) \\
3(2.5) \\
4(3.3)\end{array}$ & $\ddagger(0.0017) w$ \\
\hline Honeycombing & $10(7.0)$ & $0(0)$ & $10(8.3)$ & $\begin{array}{l}\text { N.S. } \\
(0.1511)^{x}\end{array}$ \\
\hline Normal lung field $<50 \%$ of total lung field & $12(8.4)$ & $1(4.3)$ & $11(9.1)$ & $\begin{array}{l}\text { N.S. } \\
(0.4451) \times\end{array}$ \\
\hline Emphysema & $69(47.9)$ & $6(26.1)$ & $63(52.1)$ & \\
\hline $\begin{array}{l}\text { Mild } \\
\text { - Moderate } \\
\text { - Severe } \\
\text { - Unknown }\end{array}$ & $\begin{array}{l}35(24.3) \\
26(18.1) \\
8(5.6) \\
2(1.4)\end{array}$ & $\begin{array}{l}4(17.4) \\
2(8.7) \\
0(0) \\
0(0)\end{array}$ & $\begin{array}{l}31(25.6) \\
24(19.8) \\
8(6.6) \\
2(1.7)\end{array}$ & $\doteqdot(0.0139) w$ \\
\hline \multicolumn{5}{|c|}{ Laboratory values at onset of nivolumab-associated ILD } \\
\hline WBC $\left(10^{3} / \mu \mathrm{L}\right)$ & $10.3 \pm 7.58$ & $9.12 \pm 5.34$ & $10.5 \pm 7.94$ & $\begin{array}{l}\text { N.S. } \\
(0.4091)^{t}\end{array}$ \\
\hline $\mathrm{CRP}(\mathrm{mg} / \mathrm{dL})$ & $8.62 \pm 7.66$ & $6.73 \pm 6.27$ & $8.97 \pm 7.87$ & $\begin{array}{l}\text { N.S. } \\
(0.2111)^{t}\end{array}$ \\
\hline $\mathrm{KL}-6(\mathrm{U} / \mathrm{ml})$ & $1143 \pm 1210$ & $1044 \pm 937$ & $1160 \pm 1254$ & $\begin{array}{l}\text { N.S. } \\
(0.6994)^{t}\end{array}$ \\
\hline \multicolumn{5}{|c|}{ CT findings at onset of nivolumab-associated ILD } \\
\hline Ground glass opacity & $141(97.9)$ & $23(100)$ & $118(97.5)$ & \\
\hline Consolidation & $84(58.3)$ & $12(52.2)$ & $72(59.5)$ & \\
\hline Reticulation & $34(23.6)$ & $5(21.7)$ & $29(24.0)$ & \\
\hline $\begin{array}{l}\text { Distribution } \\
\text { - Bilateral } \\
\text { - Contralateral to the tumor } \\
\text { - Ipsilateral to the tumor } \\
\text { - Not applicable }{ }^{\dagger}\end{array}$ & $\begin{array}{l}83(57.6) \\
28(19.4) \\
23(16.0) \\
10(6.9)\end{array}$ & $\begin{array}{l}15(65.2) \\
3(13.0) \\
5(21.7) \\
0(0)\end{array}$ & $\begin{array}{l}68(56.2) \\
25(20.7) \\
18(14.9) \\
10(8.3)\end{array}$ & \\
\hline \multicolumn{5}{|l|}{ Overall pattern of ILD } \\
\hline AIP/DAD-like pattern & $19(13.2)$ & $1(4.3)$ & $18(14.9)$ & $\begin{array}{l}\text { N.S. } \\
(0.5073) \times\end{array}$ \\
\hline HP-like pattern & $35(24.3)$ & $5(21.7)$ & $30(24.8)$ & \\
\hline COP-like pattern & $68(47.2)$ & $12(52.2)$ & $56(46.3)$ & \\
\hline NSIP-like pattern & $12(8.3)$ & $2(8.7)$ & $10(8.3)$ & \\
\hline Others & $10(6.9)$ & $3(13.0)$ & $7(5.8)$ & \\
\hline \multicolumn{5}{|c|}{$\begin{array}{l}\text { Data are } n(\%) \text {. } \\
\dagger \text { No tumors in any lung lobes. } \\
\ddagger p<0.05 \text {, PTI vs Non-PTI t: t-test w: Wilcoxon rank-sum test X: } \chi^{2} \text { test. } \\
\text { AIP/DAD: Acute interstitial pneumonia/diffuse alveolar damage; COP: Cryptogenic organizing pneumonia; HP: Hypersensitivity pneumonitis; ILD: Interstitial lung disease; } \\
\text { N.S.: Not statistically significant; NSIP: Nonspecific interstitial pneumonia; PTI: Peritumoral infiltration. }\end{array}$} \\
\hline
\end{tabular}

Clinical and radiological parameters were evaluated for the overall study population and for the with/without PTI subgroups. There were no notable differences in general patient characteristics between subgroups (Table 1).

Table 2 summarizes chest CT findings before nivolumab treatment and at the time of onset of nivolumabassociated pneumonitis, along with the overall patterns of ILD. Approximately a third of patients $(\mathrm{N}=52)$ had evidence of IP before nivolumab treatment, generally described as mild. The proportion of patients with no IP was numerically higher in the PTI than in the non-PTI subgroup (91.3 vs 59.2\%). At the onset of nivolumab-associated pneumonitis, the most common CT findings overall and across the two subgroups, were GGO and consolidation. Findings were mostly distributed across both lung fields. The most common ILD pattern in the overall study 


\begin{tabular}{|c|c|c|c|c|}
\hline & Total N = 144 & PTI N = 23 & Non-PTI N = 121 & $\begin{array}{l}\text { Significant difference } \\
\text { (p-value) }\end{array}$ \\
\hline \multicolumn{5}{|l|}{ Pneumonitis outcome } \\
\hline Resolved/Resolving & $110(76.4)$ & $22(95.7)$ & $88(72.7)$ & ${ }^{\dagger}(0.0171)^{w}$ \\
\hline Unchanged & $10(6.9)$ & $1(4.3)$ & $9(7.4)$ & \\
\hline Exacerbated & $23(16.0)$ & $0(0)$ & $23(19.0)$ & \\
\hline Unknown & $1(0.7)$ & $0(0)$ & $1(0.8)$ & \\
\hline ILD-associated death & $25(17.4)$ & $1(4.3)$ & $24(19.8)$ & N.S. $(0.0723)^{x}$ \\
\hline \multicolumn{5}{|c|}{ Tumor response to nivolumab treatment } \\
\hline Complete response & $0(0)$ & $0(0)$ & $0(0)$ & ${ }^{\dagger}(0.0025)^{w}$ \\
\hline Partial response & $41(28.5)$ & $12(52.2)$ & $29(24.0)$ & \\
\hline Stable disease & $49(34.0)$ & $9(39.1)$ & $40(33.1)$ & \\
\hline Disease progression & $36(25.0)$ & $1(4.3)$ & $35(28.9)$ & \\
\hline Unknown/not determined & $18(12.5)$ & $1(4.3)$ & $17(14.0)$ & \\
\hline \multicolumn{5}{|c|}{$\begin{array}{l}\text { Data are } n(\%) \text {. } \\
{ }^{\dagger} p<0.05 \text {, PTI vs non-PTI w: Wilcoxon rank-sum test } X: x^{2} \text { test. } \\
\text { ILD: Interstitial lung disease; N.S.: Not statistically significant; PTI: Peritumoral infiltration. }\end{array}$} \\
\hline
\end{tabular}

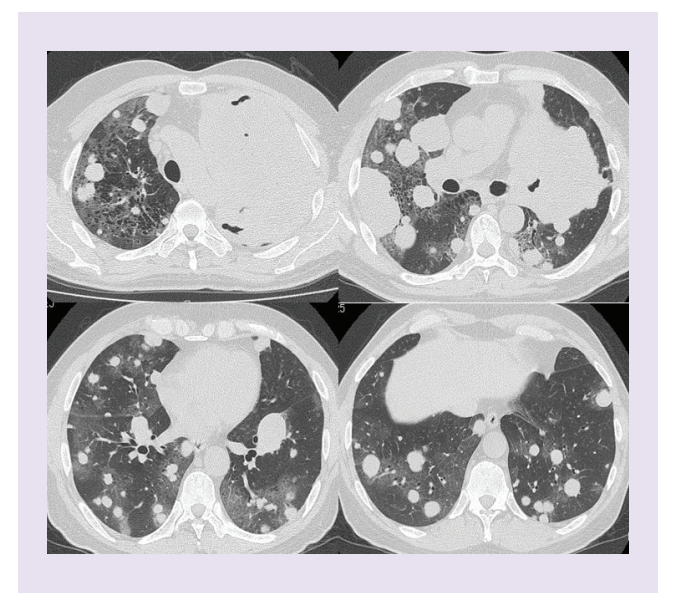

Figure 3. Fatal case of peritumoral infiltration. Fifty-nine year-old male with squamous cell lung carcinoma. Five days after initial nivolumab treatment, ground glass opacities appeared around the tumor. Peritumoral infiltration surrounding large and multiple tumors caused acute respiratory failure and death.

population was COP. Images consistent with AIP/DAD were identified in $13.2 \%$ of 144 patients and one of 23 patients (4.3\%) with PTI.

BAL was performed in 15 cases. The median proportion of lymphocytes in the BAL fluid was $35.8 \%$ (range: $1.3-76.8 \%)$.

Of 121 patients without PTI, 114 were treated with corticosteroids, while seven did not receive corticosteroids. Nivolumab was discontinued in all patients with PTI and they received corticosteroids. The outcome of pneumonitis was reported as resolved/resolving for $76.4 \%$ of patients overall, with a numerically higher proportion reported for patients with PTI (95.7\%) than for those without PTI (72.7\%) (Table 3). Fatality rates due to ILD were 1/23 (4.3\%) for the PTI subgroup (Figure 3) and 24/121 (19.8\%) for the non-PTI subgroup. Thirteen of the 19 patients presenting with an AIP/DAD like pattern died in association with ILD (all without PTI).

The tumor response to nivolumab treatment among patients with pneumonitis is summarized in Table 3 . The rate of disease progression was numerically lower in the subgroup with PTI compared with the non-PTI subgroup.

\section{Discussion}

This analysis of PMS data found that patients treated with nivolumab had observed cases of pneumonitis with PTI and showed patterns similar to those seen with exacerbation of radiation fibrosis and intensified infection, and abnormal opacities almost completely confined to the lung ipsilateral to the tumor, in addition to the typical ILD pattern observed with conventional cytotoxic chemotherapy and EGFR-TKIs. 
PTI was characterized as increased GGO that developed in a short period of time and responded well to steroids. Although pathological evidence was not available in this study, lymphocytosis in BAL fluid and good response to steroids indicate an immune response mainly consisting of lymphocyte infiltration, as described previously [2]. Because patients with PTI had a good antitumor response to nivolumab compared with non-PTI patients, PTI development may involve an antitumor immune response. A relationship between the antitumor effect of nivolumab and vitiligo associated with nivolumab treatment has been reported in melanoma patients [12]. In patients with melanoma, it has been reported that increased peritumoral PD-1 expression and immune cell infiltration at pre, on- and post-administration of nivolumab were associated with the antitumor effect [13]. In another report, biopsy of lung consolidations surrounding the tumor in a patient with metastatic melanoma to the lung detected pathological organizing pneumonia; moreover, the tumor was reduced after nivolumab treatment [14], signaling an immunological response.

Radiation pneumonitis generally occurs within 6 months of completing irradiation treatment [15], subsiding spontaneously or after steroid therapy, resulting in a fibrous lesion with collapse. Some anticancer drugs trigger the relapse of radiation pneumonitis after it has subsided, so-called 'radiation recall pneumonitis' [16]. In the current study, opacity was observed after nivolumab treatment in an area consistent with the radiation field. This radiation recall pneumonitis improved immediately upon steroid therapy, indicating immune cell infiltration. Some patients developed nivolumab-induced opacities in the radiated chest fields at 50 months after completing irradiation (compared with the usual 6-month time frame), suggesting that this may be a nivolumab-specific phenomenon [17]. Since recent advances in radiotherapy have enabled multidirectional irradiation, prior radiotherapy may complicate the assessment of nivolumab-induced pneumonitis.

Nivolumab aggravated existing nontuberculous mycobacteriosis or $P$. aeruginosa infection and may have caused inflammation of subclinical PCP infection in some patients. In the HIV setting, infection immunity that has recovered after HIV treatment can cause immune reconstitution inflammatory syndrome (IRIS) associated with occult infections with organisms such as Pneumocystis jirovecii [18]. As nivolumab activates tumor immunity by inhibiting lymphocyte PD-1, it may also activate infection immunity simultaneously; the aggravation of occult infection observed in the current study is likely to be a similar response to that seen in IRIS. A similar phenomenon can occur in HIV-negative immunodeficient patients [19]. Although this pathology is not usually classified as druginduced pneumonia, it was included in our report because of difficulty in distinguishing intensified infection from drug-induced pneumonia, and also from a drug safety perspective, as it resulted in death in a few cases.

When chemotherapy-induced pneumonia occurs asymmetrically, it is generally confined to the lung contralateral to the tumor. Although the mechanism is unknown, a relationship with the mobility of the chest wall is suggested [20]. In this study, opacity confined to the ipsilateral lung was observed after nivolumab treatment. This may indicate that PTI appears extensively in the ipsilateral lung, ipsilateral intralobar metastasis that is not revealed on CT, or carcinomatous lymphangitis.

CT image patterns consistent with AIP/DAD were identified in 14.9 and $4.3 \%$ of patients without and with PTI, respectively. Two-thirds of the patients presenting with an AIP/DAD-like pattern died in association with ILD. Such patients require careful monitoring because they have a higher risk of death than patients with other ILD patterns.

In the present study, $17.4 \%$ of patients died of nivolumab-associated ILD, a higher proportion than that reported by Delaunay et al. (9.4\%) [2]. The relatively lower incidence of ILD reported in preceding studies may be attributed to the fact that most studies analyzed patients with preserved performance status in clinical trial settings, with many clinical studies excluding patients with underlying ILD. In the present study, CT performed prior to nivolumab treatment detected ILD in $32.7 \%$ of patients. Underlying ILD is known to increase the risk of ILD-associated death in gefitinib-treated patients [21] and may also be the case in nivolumab-treated patients. Analysis of the remaining cases of pneumonitis identified during PMS is ongoing and risk factors for pneumonitis and death after the onset of pneumonitis will be reported elsewhere.

This analysis has several limitations. Although data came from an all-patient survey using a specific case report form, the survey was based on spontaneous reporting by attending physicians and did not necessarily include all patients who experienced pneumonitis. ILD was assessed using chest CT imaging and no pathological evidence was available. Tumor response data were only collected and evaluated by the ECRC from chest CT images, and not evaluated based on RECIST guidelines. Recently, combination therapy with immune checkpoint inhibitors and cytotoxic chemotherapy, or two types of immune checkpoint inhibitors, has been approved, but this analysis did not include such patients. 


\section{Conclusion}

Images of nivolumab-induced ILD showed atypical ILD patterns not observed with previous systemic therapy, which included PTI, changes similar to those seen with exacerbation of radiation-induced fibrosis and intensified infection, and abnormal opacities confined to the same lung as the tumor. PTI showed a good response to steroid treatment, suggesting that immunopotentiation is a potential cause of immune checkpoint inhibitor-associated pneumonitis. Clinicians should look for features unique to nivolumab-associated pneumonitis, in addition to the typical features of conventional drug-induced pneumonitis.

\section{Summary points}

- A new type of pneumonitis associated with immune checkpoint inhibitors, such as nivolumab, has recently been reported. However, these previous reports covered a small number of selected patients, including those without comorbidities and with good performance status.

- We assessed the clinical features and imaging characteristics of pneumonitis reported during 'real world' nationwide postmarketing surveillance of nivolumab in Japan.

- Among 144 cases analyzed, 91 (63.2\%) had radiological patterns considered typical for drug-induced pneumonitis (i.e., commonly observed during treatment with conventional chemotherapy or molecular-targeted drugs: ground glass opacity [GGO] or consolidation showing nonsegmental distribution bilaterally or dominant in the lung contralateral to the tumor).

- However, the other 53 (36.8\%) patients had previously unobserved patterns that included one or more atypical features: GGO confined to the area around the tumor, named 'peritumoral infiltration' (PTI: 23 cases [16.0\%]), intensified infection (four [2.8\%]), exacerbation of radiation fibrosis (23 [16.0\%]) and abnormal opacities largely confined to the ipsilateral lung (23 [16.0\%]).

- Patients with PTI showed a good response to corticosteroid treatment. The outcome of pneumonitis was reported as resolved/resolving for $76.4 \%$ of patients overall (PTI: $95.7 \%$; without PTI: $72.7 \%$ ).

- A higher proportion of patients with PTI than without PTI had a favorable antitumor response to nivolumab.

- In summary, images of nivolumab-induced pneumonitis showed previously unobserved radiological patterns. PTI may indicate a response to the tumor.

\section{Acknowledgments}

The authors thank the patients and their families involved in the surveillance study, and the physicians who cooperated with this analysis. Support for data analysis was provided by Micron Inc.

\section{Competing \& financial interests disclosure}

This work was supported by Ono Pharmaceutical Co., Ltd, and Bristol-Myers Squibb, Japan. No grant number is applicable.T Baba received personal fees from Ono Pharmaceutical Co., Ltd and Bristol Myers Squibb K.K. during the conduct of the study; and personal fees from AstraZeneca K.K., Boston Scientific Japan Co., Ltd, Nippon Boehringer Ingelheim Co., Ltd, Daiichi Sankyo Co., Ltd, Toray Industries Inc., Shionogi \& Co., Ltd, Astellas Pharma Inc., AMCO Inc. and Asahi Kasei Pharma Corporation, outside the submitted work. F Sakai received personal fees from Ono Pharmaceutical Co., Ltd, AstraZeneca Co., Ltd, Boehringer Ingelheim Co., Ltd, Bristol Myers Squibb K.K. and Merck Serono, during the conduct of the study; and grants/personal fees from Bayer Co., Ltd, Eisai Co., Ltd, Shionogi Pharmaceuticals Co., Ltd, Fuji Pharmaceuticals Co., Ltd, Daiichi Pharmaceuticals, Toshiba, Japanese Ministry of Labour, Health and Welfare, and the Japanese Government of Environment, outside the submitted work. T Kato received grants/personal fees from Ono Pharmaceutical Co., Ltd and Bristol Myers Squibb K.K., during the conduct of the study; and grants/personal fees from AbbVie, Astellas, AstraZeneca, Boehringer Ingelheim, Chugai, Eli Lilly, Kyowa Kirin, Merck Sharp \& Dohme, Novartis, Pfizer, Taiho, F. Hoffmann-La Roche, Merck Serono and Sumitomo Dainippon, outside the submitted work. M Kusumoto received personal fees from Ono Pharmaceutical Co., Ltd during the conduct of the study; and personal fees from AstraZeneca K.K. and MSD K.K., outside the submitted work. H Kenmotsu received personal fees from Ono Pharmaceutical Co., Ltd, and Bristol Myers K.K., during the conduct of the study; and grants/personal fees from AstraZeneca K.K., Boehringer Ingelheim, Chugai Pharmaceutical Co., Ltd, Eli Lilly K.K., Kyowa Hakko Kirin Co., Ltd, MSD K.K., Novartis Pharma K.K. and Taiho Pharmaceutical Co., Ltd, outside the submitted work. H Sugiura received personal fees from Ono Pharmaceutical Co., Ltd and MSD K.K., outside the submitted work. J Tominaga received personal fees from Ono Pharmaceutical Co., Ltd outside the submitted work. K Oikado received personal fees from Ono Pharmaceutical Co., Ltd during the conduct of the study. M Sata received personal fees from Ono Pharmaceutical Co., Ltd during the conduct of the study. M Endo received personal fees from Ono Pharmaceutical Co., Ltd during the conduct of the study. N Yanagawa received personal fees from Ono Pharmaceutical Co., Ltd during the conduct of 
the study. S Sasaki reports no conflicts of interest. T Iwasawa received personal fees from Ono Pharmaceutical Co., Ltd during the conduct of the study; and personal fees/grant from Shionogi \& Co., Ltd, Toshiba Medical System Corporation, Nihon Medi-Physics Co., Ltd, AstraZeneca K.K., Boehringer Ingelheim Japan, Inc., Bayer, GE Healthcare Japan, Tuchiya Foundation and KAKENHI, outside the submitted work. Y Saito has received honoraria for consultancy services or lectures from Ono Pharmaceutical Co., Ltd, Boehringer Ingelheim Co., Ltd, Novartis Pharma K.K., AstraZeneca K.K., MSD K.K. and Chugai Pharma Co., Ltd. Y Fujiwara received grants/personal fees from AbbVie, AstraZeneca, Chugai, Daiichi-Sankyo, Eisai, Eli Lilly, Incyte, Merck Serono, MSD, Novartis, Taiho, BMS, Ono Pharmaceutical Co., Ltd, outside the submitted work. Y Ohe received grants/personal fees from Ono Pharmaceutical Co., Ltd and Bristol Myers Squibb K.K., during the conduct of the study; and grants/personal fees from AstraZeneca, Chugai, Lilly, Pfizer, MSD, Novartis, Kyorin, Dainippon-Sumitomo, Daiichi-Sankyo, Nipponkataku, Boehringer Ingelheim, Bayer and Ignita, outside the submitted work. N Yamazaki received grants, personal fees and non-financial support from Ono Pharmaceutical Co., Ltd, during the conduct of the study; and grants/personal fees from Bristol-Myers Squibb, and Novartis Pharma K.K., outside the submitted work. T Sakamoto and T Koshiba are employees of Ono Pharmaceutical Co., Ltd. K Kuwano received personal fees from Ono Pharmaceutical Co., Ltd, during the conduct of the study. The authors have no other relevant affiliations or financial involvement with any organization or entity with a financial interest in or financial conflict with the subject matter or materials discussed in the manuscript apart from those disclosed.

Writing assistance was utilized in the production of this manuscript. Editorial support was provided by David P, Figgitt, PhD, ISMPP CMPPTM, Content Ed Net, funded by Ono Pharmaceutical Co., Ltd and Bristol-Myers Squibb, Japan.

\section{Open access}

This work is licensed under the Attribution-NonCommercial-NoDerivatives 4.0 Unported License. To view a copy of this license, visit http://creativecommons.org/licenses/by-nc-nd/4.0/

\section{References}

Papers of special note have been highlighted as: $\bullet$ of interest; $\bullet \bullet$ of considerable interest

1. Camus P, Kudoh S, Ebina M. Interstitial lung disease associated with drug therapy. Br. J. Cancer 91(Suppl. 2), S18-S23 (2004).

2. Delaunay M, Cadranel J, Lusque A et al. Immune-checkpoint inhibitors associated with interstitial lung disease in cancer patients. Eur. Respir. J. 50(2), 1700050 (2017).

3. Brahmer J, Reckamp KL, Baas P et al. Nivolumab versus docetaxel in advanced squamous-cell non-small-cell lung cancer. N. Engl. J. Med. 373(2), 123-135 (2015).

- A randomized, open-label, international Phase III study comparing nivolumab with docetaxel in previously treated patients with advanced squamous NSCLC.

4. Borghaei H, Paz-Ares L, Horn L et al. Nivolumab versus docetaxel in advanced nonsquamous non-small-cell lung cancer. N. Engl. J. Med. 373(17), 1627-1639 (2015).

- A randomized, open-label, international Phase III study comparing nivolumab with docetaxel in previously treated patients with advanced nonsquamous non-small-cell lung cancer (NSCLC).

5. Khunger M, Rakshit S, Pasupuleti V et al. Incidence of pneumonitis with use of programmed death 1 and programmed death-ligand 1 inhibitors in non-small cell lung cancer: a systematic review and meta-analysis of trials. Chest 152(2), 271-281 (2017).

•• A systematic review and meta-analysis of the incidence of pneumonitis in patients with NSCLC treated with PD-1 and PD-L1 inhibitors.

6. Nishino M, Giobbie-Hurder A, Hatabu H, Ramaiya NH, Hodi FS. Incidence of programmed cell death 1 inhibitor-related pneumonitis in patients with advanced cancer: a systematic review and meta-analysis. JAMA Oncol. 2(12), 1607-1616 (2016).

7. Gettinger SN, Zhang X, Homer R et al. Pneumonitis in non-small cell lung cancer (NSCLC) patients treated with programmed death 1 (PD1) axis inhibitors. J. Clin. Oncol. 34(15 Suppl.), Abstract 9030 (2016).

- Early report of a new type of pneumonitis, peritumoral infiltration, associated with PD-1 inhibitors in a limited number of patients.

8. Kato T, Masuda N, Tamura T et al. Nivolumab-induced interstitial lung disease analysis of two Phase II studies patients with recurrent or advanced non-small-cell lung cancer. Lung Cancer 104, 111-118 (2017).

9. Naidoo J, Wang X, Woo KM et al. Pneumonitis in patients treated with anti-programmed death-1/programmed death ligand 1 therapy. J. Clin. Oncol. 35(7), 709-717 (2017).

10. Kubo K, Azuma A, Kanazawa M et al. Consensus statement for the diagnosis and treatment of drug-induced lung injuries. Respir. Investig. 51(4), 260-277 (2013).

11. Cleverley JR, Screaton NJ, Hiorns MP, Flint JD, Müller NL. Drug-induced lung disease: high-resolution CT and histological findings. Clin. Radiol. 57(4), 292-299 (2002). 
12. Freeman-Keller M, Kim Y, Cronin H, Richards A, Gibney G, Weber JS. Nivolumab in resected and unresectable metastatic melanoma: characteristics of immune-related adverse events and association with outcomes. Clin. Cancer Res. 22(4), 886-894 (2016).

13. Vilain RE, Menzies AM, Wilmott JS et al. Dynamic changes in PD-L1 expression and immune infiltrates early during treatment predict response to PD-1 blockade in melanoma. Clin. Cancer Res. 23(17), 5024-5033 (2017).

14. Nakashima K, Naito T, Omori S et al. Organizing pneumonia induced by nivolumab in a patient with metastatic melanoma. J. Thorac. Oncol. 11(3), 432-433 (2016).

15. Giridhar P, Mallick S, Rath GK, Julka PK. Radiation induced lung injury: prediction, assessment and management. Asian Pac. J. Cancer Prev. 16(7), 2613-2617 (2015).

16. Chiang CL, Chen YW, Wu MH, Huang HC, Tsai CM, Chiu CH. Radiation recall pneumonitis induced by epidermal growth factor receptor-tyrosine kinase inhibitor in patients with advanced nonsmall-cell lung cancer. J. Chin. Med. Assoc. 79(5), 248-255 (2016).

- A study describing the occurrence of radiation recall pneumonitis induced by EGFR-TKIs in patients with NSCLC and a history of thoracic radiotherapy.

17. Shibaki R, Akamatsu H, Fujimoto M, Koh Y, Yamamoto N. Nivolumab induced radiation recall pneumonitis after two years of radiotherapy. Ann. Oncol. 28(6), 1404-1405 (2017).

18. Gopal R, Rapaka RR, Kolls JK. Immune reconstitution inflammatory syndrome associated with pulmonary pathogens. Eur. Respir. Rev. 26(143), 160042 (2017).

19. Scharschmidt TC, Amerson EH, Rosenberg OS, Jacobs RA, McCalmont TH, Shinkai K. Immune reconstitution reactions in human immunodeficiency virus-negative patients: report of a case and review of the literature. JAMA Dermatol. 149(1), 74-78 (2013).

20. Hanaoka M, Azuma A, Inokuma $S$ et al. The Japanese Respiratory Society Committee for formulation of Consensus statement for the diagnosis and treatment of drug-induced lung injuries. Consensus statement for the diagnosis and treatment of drug-induced lung injuries (2nd Edition). Japanese Respiratory Society, Tokyo, Japan, 20 (2018).

21. Kudoh S, Kato H, Nishiwaki Y et al. Interstitial lung disease in Japanese patients with lung cancer: a cohort and nested case-control study. Am. J. Respir. Crit. Care Med. 177(12), 1348-1357 (2008). 